\title{
Evaluación de un programa piloto de intervención en adultos con sobrepeso u obesidad, en riesgo de diabetes
}

\author{
Fernando Carrasco ${ }^{1}$, Manuel Moreno ${ }^{2}$, Verónica Irribarra², \\ Lorena Rodríguez ${ }^{4}$, María Antonieta Martin 4 , \\ Alejandra Alarcón ${ }^{2 a}$, Claudio Mizón ${ }^{1}$, Constanza Echenique ${ }^{1}$, \\ Víctor Saavedra ${ }^{3}$, Tito Pizarro ${ }^{4}$, Eduardo Atalah ${ }^{1,4}$. \\ Evaluation of a pilot intervention \\ program for overweight and \\ obese adults at risk of type 2 diabetes
}

Background: The Ministry of Health of Chile and selected obesity specialized centers implemented an interdisciplinary pilot program for overweight adults at risk of diabetes to decrease the risk of type 2 diabetes (T2D) and cardiovascular risk factors (CVRF). Aim: To assess the results of this program. Patients and methods: Beneficiaries of the public primary health system aged 18-45 years, with a body mass index (BM) $25-38 \mathrm{~kg} / \mathrm{m}^{2}$ and fasting blood glucose $100-$ $125 \mathrm{mg} / \mathrm{dL}$ or with any direct family member with T2D, were recruited. During the four months of the study, they were scheduled for three physician visits, four dietitian consultations, 14 physical activity sessions and four group workshops (two with a psychologist or therapist). In fasting blood samples, at the beginning and at the fourth month, glucose, insulin and lipids were determined. The Homeostasis model assessment (HOMA) index was calculated. Results: Two hundred-seventy-six patients were recruited and 160 (141 women), completed the four months of follow up. In this subgroup, at the start and end of the intervention, a BMI equal to or greater than $30 \mathrm{~kg} / \mathrm{m}^{2}$ was observed in $69 \%$ and $52 \%$ of subjects respectively, a systolic blood pressure equal to or greater than $140 \mathrm{~mm} \mathrm{Hg}$ was observed in $24 \%$ and $6 \%$ respectively, a diastolic blood pressure equal to or greater than $90 \mathrm{~mm} \mathrm{Hg}$ was observed in $28 \%$ and $9 \%$ respectively, a blood glucose equal to or greater than $100 \mathrm{mg} / \mathrm{dL}$ was observed in $61 \%$ and $19 \%$ respectively, a plasma insulin equal to or greater than $12,5 \mu \mathrm{UI} / \mathrm{ml}$ was observed in $49 \%$ and $34 \%$ respectively and a HOMA equal to or greater than 2.5 was observed in $63 \%$ and $42 \%$ respectively (all these comparisons are significant with a $\mathrm{p} \varangle 0.05$ ). Conclusions: In those patients that completed the follow up period, this intervention induced a significant decrease of some CVRF, such as BMI, fasting glucose levels and HOMA index (Rev Méd Chile 2008; 136: 13-21).

(Key w ords: Body mass index; Diabetes mellitus, type 2; Homeostasis; Obesity)

\footnotetext{
Recibido el 23 de marzo, 2007. Aceptado el 29 de agosto, 2007.

Financiamiento: Fondo Nacional de Salud.

${ }^{1}$ Departamento de Nutrición, Facultad de Medicina, Universidad de Chile, Santiago, Chile. ${ }^{2}$ Departamento de Nutrición, Diabetes y Metabolismo, Pontificia Universidad Católica de Chile, Santiago, Chile. ${ }^{3}$ Sociedad Chilena de Obesidad. ${ }^{4}$ Ministerio de Salud, Santiago, Chile. ${ }^{\text {a Nutricionista }}$
}

Correspondencia a: Dr. Fernando Carrasco N. Independencia 1027. Santiago, Chile. Fono: 9786136. Fax: 7378778. E mail: fcarrasc@med.uchile.cl 
$\mathrm{L}$ a alta y creciente prevalencia de sobrepeso y obesidad pone en grave riesgo la salud de la población chilena. La Encuesta Nacional de Salud realizada en 2003, demostró que $61 \%$ de la población mayor de 17 años presentaba exceso de peso, proporción que era aún mayor en mujeres y en personas de mayor edad ${ }^{1}$.

Las personas obesas tienen tasas más altas de resistencia a la insulina, intolerancia a la glucosa, diabetes mellitus tipo 2 (DM2), hipertensión arterial (HTA), dislipidemia y cardiopatía coronaria, entre otras patologías, lo que afecta la calidad de vida y disminuye en 5 a 10 años la esperanza de vida $^{2-6}$. El riesgo relativo de DM2 e HTA es $\sim 3$ veces mayor en adultos obesos que en no obesos, cifra que se eleva a 3,8 y 5,6 veces, respectivamente, en el segmento de 25 a 45 años ${ }^{7}$.

La diabetes determina una importante carga económica y social para los países ${ }^{8,9}$. La DM2 es tanto un factor de riesgo como un indicador de daño cardiovascular, pues 70\% de los diabéticos muere por infarto o accidente vascular ${ }^{10}$. Se relaciona además con discapacidad e invalidez por las complicaciones derivadas de ella, como insuficiencia renal ( $40 \%$ de los pacientes que requieren diálisis son diabéticos) ${ }^{11}$. Se estima que esta enfermedad es la primera causa de ceguera y de amputaciones de extremidades en sujetos mayores de 40 años $^{12}$.

Diversos estudios indican que es posible evitar o retardar la aparición de la DM2, a través de intervenciones dirigidas a modificar los hábitos de alimentación y actividad física ${ }^{13}$. Un descenso de peso discreto ( $5 \%$ a 10\%), está asociado con una mejoría significativa en los niveles de presión arterial en individuos con o sin HTA, con un riesgo relativo (RR) de tener HTA de 0,35 , respecto al grupo control ${ }^{14}$. La disminución de peso logra además una mejoría del perfil lipídico, de la tolerancia a la glucosa y resistencia insulínica ${ }^{15,16}$. En el estudio de prevención de diabetes en Finlandia, en 552 sujetos con intolerancia a la glucosa, la incidencia de diabetes disminuyó en $58 \%$ al cabo de 3,2 años de seguimiento en el grupo intervenido con un programa de actividad física y alimentación saludable ${ }^{17}$. En el estudio de prevención de diabetes de Estados Unidos de Norteamérica, en 3.234 sujetos con intolerancia a la glucosa, después de 2,8 años de seguimiento, se observó que la intervención con cambios en estilo de vida logró una reducción en la incidencia de DM2 de 58\% en comparación con placebo ${ }^{18,19}$.

Con estos antecedentes FONASA (Fondo Nacional de Salud) y el Ministerio de Salud planificaron un programa piloto en personas con sobrepeso y alto riesgo de desarrollar DM2, con el objetivo de reducir el peso corporal y la resistencia insulínica y prevenir la aparición de DM2. La intervención se basó en evaluaciones y controles médicos y de nutricionista, actividades educativas individuales y grupales, consistentes en cambios en la dieta, apoyo por terapeuta ocupacional o psicólogo y ejercicio. El propósito de este análisis es evaluar el impacto de este programa y aportar antecedentes para su eventual expansión en el sistema público y privado, por la modalidad FONASA libre elección e institucional.

\section{Material y MÉTOdo}

El proyecto consistió en promover estilos de vida saludables en adultos con sobrepeso u obesidad en riesgo de diabetes, beneficiarios del sistema público de salud, detectados en el examen de salud preventivo del adulto $u$ otra actividad de salud. Los pacientes fueron reclutados entre diciembre de 2004 y junio de 2005 . Se definió por el grupo investigador una canasta de prestaciones, las que fueron valoradas por FONASA en 88.000 pesos por persona ( 160 dólares), incluyendo las atenciones médicas, de nutricionista, sesiones grupales e individuales y los exámenes de laboratorio. Se excluyó en este primer proyecto todo tratamiento con fármacos anorexígenos o hipoglicemiantes. Se definió un protocolo de intervención con las distintas actividades a realizar durante 6 meses de seguimiento.

Criterios de ingreso. Se seleccionaron para el estudio personas entre 18 y 45 años, con un índice de masa corporal (IMC) entre 25 y $38 \mathrm{~kg} / \mathrm{m}^{2}$, con antecedentes de familiar de primer grado con DM2 o con glicemia en ayunas entre 100 y 125 mg/dL. Los individuos fueron detectados en sus centros de Atención Primaria y derivados a los centros participantes en el estudio para su ingreso al programa de intervención. Se excluyeron sujetos con antecedentes de eventos cardiovasculares (infarto agudo al miocardio, accidente vascular encefálico u otro semejante), insuficiencia 
hepática, cardiaca o renal crónica, patología oncológica o de cualquier otra patología que representara contraindicación para someterse al plan de intervención. Ingresaron al estudio pacientes con hipertensión arterial controlada. El programa de intervención y su consentimiento informado fue sometido a la aprobación de los comités de ética respectivos a cada centro participante en el estudio. A todos los pacientes se les explicó los detalles del programa luego de lo cual firmaron un formulario de consentimiento informado.

Las intervenciones profesionales y exámenes realizados fueron libres de costo para los pacientes.

Diseño. Corresponde a un estudio multicéntrico, prospectivo, no controlado, en el cual participaron 4 centros: Departamento de Nutrición, Pontificia Universidad Católica de Chile; Departamento de Nutrición, Universidad de Chile; Sociedad Chilena de Obesidad y Centro de Salud Familiar (CESFAM) "Orlando Letelier", Servicio de Salud Metropolitano Sur.

Programa de intervención. El programa consideró 4 meses de intervención, con un control tardío dos meses después. El plan de atención multiprofesional incluyó:

- Atenciones médicas: al inicio, al cuarto y sexto mes. En las sesiones médicas se efectuó anamnesis y examen físico, registro de peso y circunferencia de cintura, diagnóstico nutricional y de enfermedades asociadas, cálculo de requerimientos y prescripción de plan alimentario para reducción de peso. La indicación de energía se calculó para obtener un balance energético de 500 a $700 \mathrm{kcal} /$ día bajo las necesidades de mantención, con un aporte mínimo de 1.200 $\mathrm{kcal} /$ día, con una contribución de las proteínas al aporte energético de $20 \%$, fraccionada en al menos 3 comidas y 2 colaciones.

- Atenciones de nutricionista: al inicio, a los 15 días, al segundo y tercer mes. En estas sesiones se realizó encuesta alimentaria con aplicación de cuestionario de frecuencia de consumo, se desarrolló plan de alimentación individualizado, se efectuó educación alimentaria y refuerzo de las indicaciones nutricionales.

Durante las visitas con médico y nutricionistas se realizaron en condiciones estandarizadas controles de peso, circunferencia de cintura y presión arterial.
- Sesiones de actividad física, supervisadas por profesor de educación física o kinesiólogo: 2 sesiones semanales el primer mes, 1 sesión semanal el segundo mes y 2 sesiones el tercer mes. El objetivo fue desarrollar un plan de actividad física el que tuvo una fracción supervisada y una fracción no supervisada. La actividad a desarrollar fue de tipo aeróbica, de intensidad moderada y de duración mínima efectiva de $30 \mathrm{~min}$ por sesión, continua o intermitente. En la primera y última sesión se aplicó un test breve de evaluación cardiovascular (test de marcha de 6 min). Cada paciente recibió una cartilla educativa sobre los beneficios de realizar actividad física y actividades prácticas propuestas para el hogar, lugar de trabajo o ambos. Se emitió un informe de cada paciente al final del tercer mes, con los resultados de esta actividad.

- Sesiones educativas grupales de una hora aproximadamente, con un número máximo de 10 personas por grupo: 2 consultas por psicólogo o terapeuta durante el segundo y tercer mes, una atención por médico al primer mes y una por nutricionista al cuarto mes. En la sesión grupal médica se abordó la definición de obesidad, sus principales factores predisponentes, los riesgos asociados al exceso de peso y la posibilidad de controlar esta condición mediante un cambio en los hábitos de alimentación y de actividad física. Durante el taller grupal con nutricionista, los principales temas tratados fueron alimentación saludable, guías y pirámide alimentaria, etiquetado nutricional y cóctel saludable. En las sesiones grupales con psicólogo o terapeuta, los objetivos fueron: favorecer la capacidad de reconocer y descubrir el sentido y significado personal de los hábitos alimentarios, en un contexto históricofamiliar transgeneracional; favorecer la toma de conciencia acerca de los factores ambientales, emocionales y relacionales involucrados en la conducta alimentaria y facilitar un proceso de reflexión, evaluación y autocrítica que promueva un cambio hacia un estilo de vida más saludable.

Exámenes de laboratorio. Al inicio y a los 4 meses de seguimiento se determinó, en condiciones de ayuno, glicemia, insulinemia y perfil lipídico. Se 
calculó el índice de resistencia insulínica HOMA con la siguiente fórmula: (glicemia $\mathrm{mg} / \mathrm{dL} \mathrm{x}$ insulinemia $\mu \mathrm{U} / \mathrm{mL}$ ) $/ 405^{20}$. Se consideraron alterados los siguientes valores: glicemia $\geq 100 \mathrm{mg} / \mathrm{dL}$, insulinemia $\geq 12,5 \mu \mathrm{U} / \mathrm{mL}$, HOMA $\geq 2,5$, colesterol total $\geq 200 \mathrm{mg} / \mathrm{dL}$, colesterol HDL $\leq 45 \mathrm{mg} / \mathrm{dL}$, colesterol LDL $\geq 130 \mathrm{mg} / \mathrm{dL}$ y triglicéridos $\geq 150 \mathrm{mg} / \mathrm{dL}$.

Estadística. Se analizó la normalidad de cada variable a través del test de Shapiro-Wilk. Dado que la mayor parte de las variables no tuvieron una distribución normal, se trabajó con medianas y percentiles como medidas de tendencia central y con el test de Kruskal Wallis para la comparación entre los grupos. Para analizar los cambios intragrupo por efecto de la intervención se usó la prueba Signrank test de Wilcoxon, considerando sólo a los pacientes que completaron el plan de tratamiento. En las variables categóricas se utilizaron distribuciones de frecuencia y el test de chi cuadrado para la comparación entre los grupos. Las variables analizadas fueron la mediana de los indicadores antropométricos y bioquímicos entre el ingreso y el control de los 4 meses. Se analizaron, además, los cambios en la prevalencia de indicadores bioquímicos y cifras de presión arterial fuera del rango normal. Se consideró significativo un $\mathrm{p}<0,05$. Todos los análisis se realizaron con el programa estadístico STATA ${ }^{\mathrm{TM}}$, versión 9.2.

\section{Resultados}

Ingresaron al estudio 276 pacientes ( 35 hombres y 241 mujeres) cuyas características generales se presentan en la Tabla 1. La edad promedio de los hombres fue mayor que la de las mujeres ( $p$ $<0,05)$. Del total de la muestra, $50 \%$ presentaba obesidad moderada (IMC entre 30 y $34,9 \mathrm{~kg} / \mathrm{m}^{2}$ ), $25 \%$ sobrepeso (IMC entre 25 y $29,9 \mathrm{~kg} / \mathrm{m}^{2}$ ), y $25 \%$ obesidad severa (IMC $\geq 35 \mathrm{~kg} / \mathrm{m}^{2}$ ). La circunferencia de cintura se presentó en rango de obesidad abdominal en $84,2 \%$ de los casos. Los niveles de glicemia de ayunas, insulinemia basal y HOMA se encontraban aumentados al ingreso en $57,4 \%, 53,4 \%$ y $65,1 \%$ de los sujetos, respectivamente. El colesterol de LDL, colesterol de HDL y triglicéridos plasmáticos se encontraban en niveles

Tabla 1. C aracterísticas generales del grupo intervenido, según sexo

\begin{tabular}{|c|c|c|c|c|c|c|c|}
\hline \multirow[t]{2}{*}{ Variable } & \multicolumn{3}{|c|}{$\begin{array}{c}\text { Hombres } \\
\mathrm{n}=35\end{array}$} & \multicolumn{3}{|c|}{$\begin{array}{l}\text { Mujeres } \\
\mathrm{n}=241\end{array}$} & \multirow[t]{2}{*}{$\mathrm{P}$} \\
\hline & p50 & p25 & p75 & $\mathrm{p} 50$ & p25 & p75 & \\
\hline Edad (años) & 41 & 35 & 43 & 37 & 29 & 42 & $<0,05$ \\
\hline Talla (m) & 1,73 & 1,68 & 1,75 & 1,57 & 1,53 & 1,61 & N.A. \\
\hline Peso $(\mathrm{kg})$ & 97,9 & 87,0 & 103,0 & 77,8 & 71,7 & 85,0 & N.A. \\
\hline $\operatorname{IMC}\left(\mathrm{kg} / \mathrm{m}^{2}\right)$ & 32,6 & 30,0 & 35,2 & 32,0 & 29,6 & 34,6 & N.S. \\
\hline Circunferencia cintura $(\mathrm{cm})$ & 107 & 101 & 111 & 97 & 90 & 104 & N.A \\
\hline Presión sistólica (mm Hg) & 130 & 122 & 140 & 125 & 120 & 134 & N.S. \\
\hline Presión diastólica (mm Hg) & 80 & 75 & 90 & 80 & 75 & 90 & N.S. \\
\hline Glicemia en ayuno $(\mathrm{mg} / \mathrm{dL})$ & 102 & 88 & 108 & 100 & 90 & 107 & N.S. \\
\hline Insulinemia basal (mU/mL) & 13,7 & 9,4 & 19,2 & 12,8 & 8,6 & 18,6 & N.S. \\
\hline HOMA & 3,0 & 2,1 & 4,7 & 3,1 & 2,0 & 4,6 & N.S. \\
\hline Colesterol total (mg/dL) & 193 & 163 & 223 & 186 & 160 & 213 & N.S. \\
\hline Colesterol LDL (mg/dL) & 127 & 99 & 162 & 109 & 88 & 129 & N.S. \\
\hline Colesterol HDL (mg/dL) & 43 & 35 & 51 & 44 & 39 & 49 & N.S. \\
\hline Triglicéridos (mg/dL) & 223 & 126 & 285 & 122 & 85 & 184 & $<0,001$ \\
\hline
\end{tabular}

N.A.: no aplicable; N.S.: no significativo; IMC: índice de masa corporal; HOMA: índice de resistencia insulínica (Homeostasis Model Assessment).

Test de Kruskal Wallis para diferencia entre grupos. 
de riesgo en $26,3 \%, \quad 55,9 \%$ y $37,9 \%$ de los pacientes, respectivamente. Los hombres presentaron niveles de triglicéridos más elevados que las mujeres $(\mathrm{p}<0,001)$.

Completaron el estudio 160 pacientes (141 mujeres y 19 hombres), lo que determina una pérdida de $42 \%$ al cuarto mes de seguimiento, con un comportamiento similar en hombres y mujeres (NS). Las principales causas de abandono de los pacientes ( $\mathrm{n}$ =116), fueron la poca motivación con los resultados de la pérdida de peso (57\%) y el costo económico de trasladarse a los centros de tratamiento (27\%). En este grupo no se encontraron diferencias significativas, en comparación con los que completaron el seguimiento, en variables como edad, IMC, actividad ocupacional, actividad de tiempo libre o antecedentes familiares de DM2. Tampoco se encontró diferencia significativa en los valores iniciales de glicemia de ayunas y lípidos plasmáticos, pero los sujetos que abandonaron el programa antes del $4^{\circ}$ mes tenían cifras significativamente mayores de insulinemia basal ( $p<0,01$ ) y HOMA ( $p<0,001$ ). Al comparar los resultados entre los centros, no se evidenció diferencias significativas.

En el grupo que completó los 4 meses de intervención, se observó una reducción significativa del peso corporal (Figura 1), IMC y circunferencia de cintura. Al final del período la mediana de cambio fue de $-4,1 \mathrm{~kg},-1,8$ unidades de IMC y
$-6 \mathrm{~cm}$ de cintura, en relación al ingreso $(\mathrm{p}<0,001)$. La mediana de reducción de peso, como porcentaje del peso inicial, fue de 3,93\% en mujeres y $3,81 \%$ en hombres (3,9\% en el grupo total).

La Tabla 2 muestra los cambios en los indicadores bioquímicos. Durante el seguimiento se observó una reducción significativa de glicemia (6,5\%), insulinemia (-9,6\%), HOMA (-14,3\%) y colesterol LDL $(-2,7 \%)$, y un aumento del colesterol HDL $(6,8 \%)$. No hubo variación significativa en los niveles de triglicéridos.

En la Tabla 3 se observan los cambios en los indicadores bioquímicos de acuerdo a la magnitud de la pérdida de peso, observándose que sólo la glicemia y el HOMA muestran una mayor reducción en el grupo que perdió más de 5\% de su peso inicial.

La Tabla 4 muestra la prevalencia de indicadores alterados al inicio y al final del plan de intervención. La prevalencia de glicemia en ayuno $\geq 100 \mathrm{mg} / \mathrm{dL}$ se redujo en $68 \%$, de insulinemia $\geq 12,5 \mu \mathrm{U} / \mathrm{mL}$ en $30 \%$ y de HOMA $\geq 2,5$ en $33 \%(\mathrm{p}<0,005)$.

$\mathrm{Al}$ analizar los principales indicadores de impacto se observó que en los 160 sujetos que terminaron la intervención, 55\% logró una reducción de al menos $5 \%$ del peso inicial, 51,2\% normalizó su glicemia de ayuno y 37,3\% normalizó el índice HOMA.

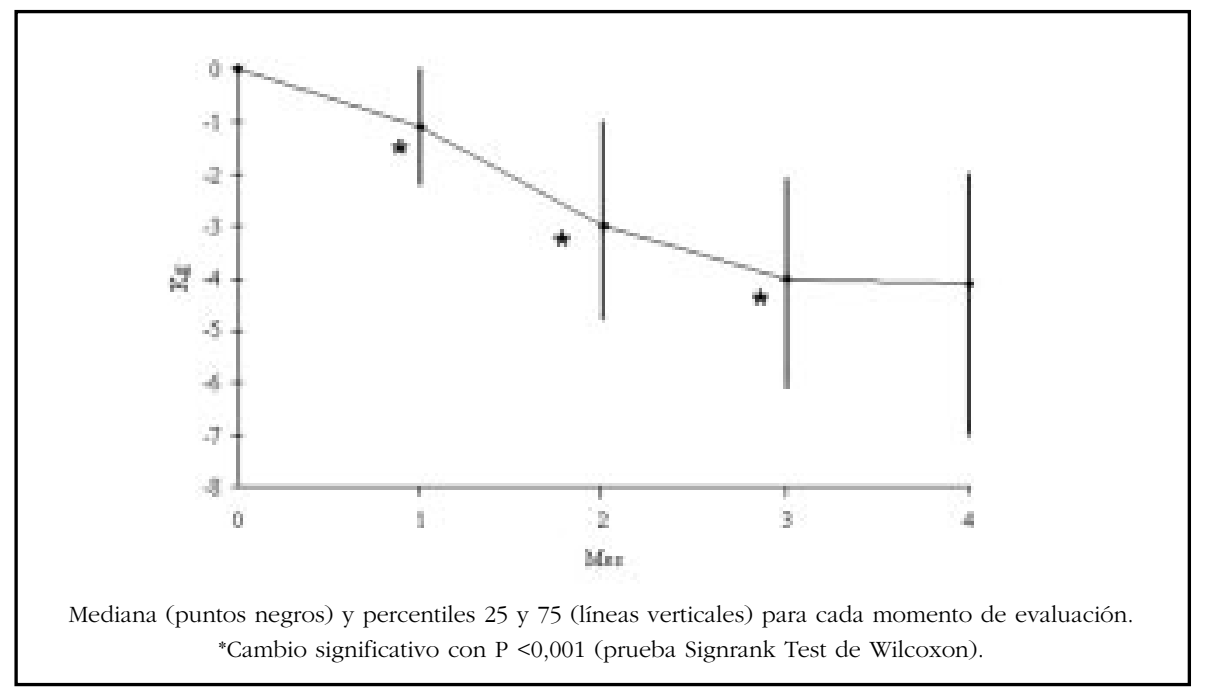

Figura 1. Cambio de peso en relación al ingreso durante los 4 meses de seguimiento. 
Tabla 2. C ambio en los indicadores bioquímicos desde el ingreso hasta los 4 meses de seguimiento

\begin{tabular}{|lrcrcc|}
\hline Variable & \multicolumn{2}{c}{ Ingreso } & \multicolumn{2}{c|}{ Final } & P \\
& \multicolumn{2}{c}{$\mathrm{n}=160$} & \multicolumn{2}{c|}{$\mathrm{n}=160$} & \\
& Mediana & $(\mathrm{p} 5 / \mathrm{p} 95)$ & Mediana & $(\mathrm{p} 5 / \mathrm{p} 95)$ & \\
\hline Glicemia en ayuno $(\mathrm{mg} / \mathrm{dL})$ & 101,0 & $(77,0 / 121,8)$ & 91,0 & $(70,5 / 112,0)$ & $<0,001$ \\
Insulinemia basal $(\mu \mathrm{U} / \mathrm{mL})$ & 11,5 & $(4,5 / 26,0)$ & 10,2 & $(3,7 / 25,0)$ & $<0,05$ \\
HOMA & 2,8 & $(1,1 / 6,8)$ & 2,3 & $(0,79 / 6,2)$ & $<0,001$ \\
Colesterol total $(\mathrm{mg} / \mathrm{dL})$ & 186,0 & $(117,2 / 270,6)$ & 181,0 & $(123,3 / 245,8)$ & N.S. \\
Colesterol LDL $(\mathrm{mg} / \mathrm{dL})$ & 112,0 & $(60,5 / 176,3)$ & 112,0 & $(53,4 / 166,9)$ & N.S. \\
Colesterol HDL $(\mathrm{mg} / \mathrm{dL})$ & 43,5 & $(30,0 / 68,0)$ & 47,0 & $(34,3 / 70,8)$ & $<0,001$ \\
Triglicéridos $(\mathrm{mg} / \mathrm{dL})$ & 117,0 & $(51,0 / 335,9)$ & 117,5 & $(57,8 / 277,8)$ & N.S. \\
\hline
\end{tabular}

p5 y p95: percentil 5 y percentil 95, respectivamente.

HOMA: índice de resistencia insulínica (Homeostasis Model Assessment).

Prueba Signrank test de Wilcoxon para cambio intragrupo.

Tabla 3. M ediana y percentil 5 y 95 de cambio en los indicadores metabólicos en función del porcentaje de cambio del peso corporal después de 4 meses de intervención

\begin{tabular}{|lccccc|}
\hline Variable & \multicolumn{2}{c}{$\geq 5 \%$ peso inicial } & \multicolumn{2}{c|}{$<5 \%$ peso inicial } & $\begin{array}{c}\mathrm{P} \\
\mathrm{n}=88\end{array}$ \\
& Mediana & $(\mathrm{p} 5 / \mathrm{p} 95)$ & Mediana & $(\mathrm{p} 5 / \mathrm{p} 95)$ & \\
\hline Glicemia $(\mathrm{mg} / \mathrm{dL})$ & $-9,5$ & $(-36,5 / 20,1)$ & $-4,0$ & $(-31,6 / 14,0)$ & $<0,05$ \\
Insulinemia basal $(\mu \mathrm{U} / \mathrm{mL})$ & $-2,0$ & $(-15,9 / 10,3)$ & $-0,3$ & $(12,6 / 15,4)$ & 0,06 \\
HOMA & $-0,6$ & $(-4,2 / 0,9)$ & $-0,1$ & $(-3,1 / 3,8)$ & $<0,05$ \\
Colesterol total $(\mathrm{mg} / \mathrm{dL})$ & $-3,0$ & $(-65,2 / 36,0)$ & $-3,0$ & $(-49,4 / 50,6)$ & N.S. \\
Colesterol HDL $(\mathrm{mg} / \mathrm{dL})$ & $+4,0$ & $(-9,3 / 16,3)$ & $+2,0$ & $(-8,6 / 14,4)$ & N.S. \\
Colesterol LDL $(\mathrm{mg} / \mathrm{dL})$ & $-2,0$ & $(-65,5 / 30,9)$ & $-6,0$ & $(-49,0 / 66,1)$ & N.S. \\
Triglicéridos $(\mathrm{mg} / \mathrm{dL})$ & $-11,0$ & $(-171,8 / 100,3)$ & $+3,0$ & $(-141,2 / 128,8)$ & N.S. \\
\hline
\end{tabular}

p5 y p95: percentil 5 y percentil 95, respectivamente.

HOMA: índice de resistencia insulínica (Homeostasis Model Assessment).

Prueba de Kruskal Wallis para diferencia entre grupos.

\section{DisCUSIÓN}

La elevada prevalencia de obesidad en nuestro país, especialmente en las poblaciones de bajo nivel socioeconómico y educacional, representa un serio problema de salud pública ${ }^{1}$, debido a su asociación con enfermedades cardiovasculares y diabetes, que a su vez generan un alto impacto en morbimortalidad y costos en salud. Existen múltiples evidencias que demuestran que la disminu- ción de peso se asocia a la prevención de diabetes en individuos obesos con intolerancia a la gluco$\mathrm{sa}^{17-19}$. Nuestro país no cuenta con programas preventivos que consideren el financiamiento de actividades que fomentan cambios en hábitos de alimentación y de actividad física. La única posibilidad actual de las personas en riesgo de diabetes, es realizarse los controles periódicos de salud e incorporarse a un programa de intervención cuando la diabetes ya se ha diagnosticado. 
Tabla 4. Prevalencia de obesidad y trastornos metabólicos al inicio y después de 4 meses de intervención

\begin{tabular}{|lccc|}
\hline Variable & $\begin{array}{c}\text { Inicial }(\mathrm{n}=160) \\
\%\end{array}$ & $\begin{array}{c}\text { Final }(\mathrm{n}=160) \\
\%\end{array}$ & $\mathrm{P}$ \\
\hline & & & \\
IMC $\geq 30 \mathrm{~kg} / \mathrm{m}^{2}$ & 68,8 & 51,9 & $<0,005$ \\
Obesidad abdomina* & 87,5 & 63,8 & $<0,001$ \\
Presión sistólica $\geq 140 \mathrm{~mm} / \mathrm{Hg}$ & 24,3 & 5,7 & $<0,001$ \\
Presión diastólica $\geq 90 \mathrm{~mm} / \mathrm{Hg}$ & 28,1 & 9,4 & $<0,001$ \\
Glicemia $\geq 100 \mathrm{mg} / \mathrm{dL}$ & 60,6 & 19,2 & $<0,001$ \\
Insulinemia $\geq 12,5 \mu \mathrm{U} / \mathrm{mL}$ & 48,5 & 33,7 & $<0,005$ \\
HOMA $\geq 2,5$ & 63,0 & 42,0 & $\mathrm{NS}$ \\
Colesterol total $\geq 200 \mathrm{mg} / \mathrm{dL}$ & 37,0 & 32,0 & $\mathrm{NS}$ \\
Colesterol HDL $\geq 45 \mathrm{mg} / \mathrm{dL}$ & 49,0 & 60,2 & $\mathrm{NS}$ \\
Colesterol LDL $\geq 130 \mathrm{mg} / \mathrm{dL}$ & 30,9 & 25,7 & $\mathrm{NS}$ \\
Triglicéridos $\geq 150 \mathrm{mg} / \mathrm{dL}$ & 36,6 & 27,7 & \\
\hline
\end{tabular}

IMC: índice de masa corporal; HOMA: índice de resistencia insulínica (Homeostasis Model Assessment). *Obesidad abdominal: circunferencia de cintura $\geq 88 \mathrm{~cm}$ en mujeres y $\geq 102 \mathrm{~cm}$. en hombres.

Chi cuadrado para cambio de proporción intragrupo.

En este sentido, el sistema es más bien reactivo y diseñado para financiar atenciones curativas: atención médica, exámenes de laboratorio, hospitalizaciones, entre las más importantes; sin embargo, aquellas acciones de carácter preventivo, y de más bajo costo, no cuentan con incentivos ni el apoyo financiero necesario.

Hasta la implementación de este programa de intervención, FONASA no contaba con un programa multidisciplinario de atención integral para pacientes con sobrepeso u obesidad, debiendo éstos pagar en forma independiente cada atención $\mathrm{e}$, incluso, en forma particular las atenciones por nutricionista, recurso profesional indispensable en este tipo de tratamientos.

La percepción en el público general, y también entre los profesionales de la salud, es que muy pocos pacientes logran resultados exitosos en el control de peso a largo plazo. Si bien esa percepción tiene base en la literatura ${ }^{21}$, existen estudios que muestran que entre individuos que pierden intencionalmente al menos $10 \%$ del peso inicial y lo mantienen al menos por un año, 20\% es capaz de mantener su peso a largo plazo 22. Datos del Programa de Obesidad de la Pontificia Universidad Católica de Chile, muestran que los pacientes sometidos a un período de 4 meses de tratamiento multidisciplinario (controles de médico, nutricionista, psicólogo y kinesiólogo) logran disminuir al menos $10 \%$ del peso corporal ${ }^{23}$. Aquellos que siguen un plan de mantención, en que se refuerzan conductas, logran mantener sus objetivos de peso por más de un año. Los análisis de estudios de intervención con cambios de estilo de vida para personas prediabéticas con sobrepeso han sido exitosos, más aún si se han incluido intervenciones de tipo cognitivo conductual, como las planteadas en este proyecto ${ }^{24,25}$. Aunque en el grupo intervenido en este programa, la reducción de peso después de 4 meses fue en promedio de sólo $\sim 4 \%$ del peso inicial, se obtuvo una reducción significativa en los principales indicadores bioquímicos de impacto, tales como la glicemia de ayunas y la insulinemia basal, con un bajo costo considerando la complejidad y duración de la intervención. Sin bien el programa aplicado en la población analizada incluye los elementos de intervención multiprofesional adecuados para un cambio en el estilo de vida, algunos factores de vulnerabilidad socioeconómica pudieran haber influido negativamente en el cumplimiento de las recomendaciones de dieta y ejercicio físico. Los estudios exitosos de intervención no farmacológica en sujetos con sobrepeso y 
riesgo de diabetes basan su eficacia, al menos parcialmente, en la mantención de los cambios conductuales tanto en el ámbito familiar, como en el social y laboral. Aun así, de los pacientes que completaron el programa de intervención, 55\% logró una pérdida de al menos 5\% del peso inicial, en comparación con el 43\% observado durante el primer año de intervención en 265 sujetos prediabéticos finlandeses, quienes recibieron un fuerte apoyo social y laboral orientado a un cambio perdurable en su estilo de vida ${ }^{17}$.

La implementación de este tipo de intervención en individuos con sobrepeso y factores de riesgo de diabetes (ej: con glicemia de ayuno alterada o riesgo familiar de diabetes), tiene amplias perspectivas de resultados favorables, pues plantea una acción de índole preventiva. El proyecto está enfocado en el segmento de población atendida en consultorios, que es la más afectada por sobrepeso y obesidad ${ }^{1}$, lo que permitirá reproducir ampliamente sus resultados en toda la población, contribuyendo a disminuir la inequidad en salud. Un hecho que refuerza esta idea, fue que un centro de atención de este proyecto piloto fue un CESFAM del Servicio de Salud Sur de Santiago. La aplicación masiva de este programa de intervención cumpliría además con el objetivo de contener el aumento de la obesidad y contribuir a la disminución de la mortalidad por enfermedades cardiovasculares, que son algunos de los objetivos sanitarios de salud ${ }^{26}$.

Uno de los problemas observados en esta intervención fue la alta tasa de abandono (42\%). El abandono del plan de tratamiento es uno de los principales problemas observados en estudios de manejo de obesidad. En una revisión de estudios de tratamiento no farmacológico de obesidad, con fases de reducción de peso de 2 a 12 meses, Teixeira y cols observaron que las menores tasas de abandono fueron de 50\% a 55\%, y que a menudo la causa del abandono se debía a una baja reducción de peso inicial ${ }^{27}$. Al analizar las causas de abandono en nuestra serie, se puede observar que en muchos pacientes el ambiente sociocultural del que provienen, y las dificultades

\section{REFERENCIAS}

1. Ministerio de Salud. Encuesta Nacional de Salud, 2003. Minsal, Chile. http://epi.minsal.cl/epi/html/ intrafamiliares, reducen significativamente la adherencia a un programa de esta naturaleza. Además, las expectativas de una mayor reducción de peso que la observada en los primeros meses de intervención, reducen la motivación para mantenerse en el programa. Para mejorar la efectividad de futuros programas es importante considerar la motivación, expectativas y potenciales dificultades de cada sujeto al momento de ingresar al plan de intervención. Factores tales como la motivación propia para perder peso, el apoyo social, mayor capacidad de soportar el estrés, autonomía, mayor responsabilidad y autocuidado y la mayor estabilidad psicológica, se han asociado a una mayor probabilidad de éxito en la reducción de peso y mantención de los resultados en el largo plazo $^{28}$.

Basado en los resultados de los estudios de prevención de DM2 en pacientes con sobrepeso u obesidad en Europa y Estados Unidos de Norteamérica, en la decisión ministerial de extender este programa a diferentes CESFAM del país, y en el desarrollo de un fondo por parte de FONASA para el financiamiento de un grupo de prestaciones similares a este programa en el sistema de libre elección (ej: programa de atención por nutricionistas), se hace imprescindible, desde el punto de vista de salud pública, una evaluación del impacto de este tipo de intervención, una vez que sea aplicada a un mayor porcentaje de la población en riesgo. Esto se debiera traducir en una disminución en la incidencia de obesidad y diabetes, reducción en las complicaciones cardiovasculares, mejoría en la calidad de vida y disminución de los costos en salud de nuestro país ${ }^{26}$.

Es de suma importancia evaluar los resultados que se obtengan al replicar este programa en otros CESFAM del sistema público de salud y, de acuerdo a lo que se considera un manejo óptimo de pacientes crónicos, se requiere de la implementación de programas de seguimiento de estos pacientes en el largo plazo, para reforzar las medidas nutricionales, de promoción de actividad física y los contenidos educativos entregados en esta primera etapa.

invest/ENS/InformeFinalENS.pdf. (Consultado el 20 de febrero de 2007).

2. WHO. Diet, nutrition, and the prevention of chronic diseases. Report of a WHO Study Group. 
Geneva, World Health Organization, 1990 (WHO Technical Report Series No 797).

3. WHO. Obesity. Preventing and management the global epidemic. Report of a WHO Consultation on Obesity. Geneva: WHO, 1997.

4. Drenick EJ, Bale GS, Seltzer F, Johnson DG. Excessive mortality and causes of death in morbidity obese men. JAMA 1980; 243: 443-5.

5. Olshansky SJ, Passaro DJ, Hershow RC, Layden J, Carnes BA, Brody J et al. A potential decline in the Unites States life expectancy in the 21st century. N Engl J Med 2005; 352: 1138-45.

6. Fontaine KR, Redden DT, Wang C, Westfall aO, Aluison DB. Years of life lost due to obesity. JAMA 2003; 289: 187-93.

7. Manson Je, Sherret PJ, Greenland P, Van Itallie TB. The escalating pandemics of obesity and sedentary lifestyle. Arch Intern Med 2004; 164: 249-58.

8. WHO. The Cost of Diabetes in Latin America and the Caribbean. Bulletin of The World Health Organization 2003; 81(1).

9. McQuaid S, O'Gorman DJ, Yousif O, Yeow TP, Rahman Y, Gasparro D et al. Early onset insulinresistant diabetes in obese caucasians has features of typical type 2 diabetes, but 3 decades earlier. Diabetes Care 2005; 28: 1216-9.

10. Jemal A, Ward E, Hao Y, Thun M. Trends in the leading causes of death in the United States, 1970-2002. JAMA 2005; 294: 1255-9.

11. Mezzano S, Aros CE. Enfermedad renal crónica: clasificación mecanismos de progresión y estrategias de renoprotección. Rev Méd Chile 2005; 133: 338-48.

12. Organización Panamericana de Salud. Perfil de salud de Chile. Datos actualizados para el año 2001. http://www.paho.org/Spanish/SHA/prflchi.htm

13. Klein S, Sheard NF, Pi-Sunyer X, Daly A, WylieRosett J, Kulkarni K et al. Weight management through lifestyle modification for the prevention and management of type 2 diabetes: rationale and strategies. A statement of the American Diabetes Association, the North American Association for the Study of Obesity, and the American Society for Clinical Nutrition. Am J Clin Nutr 2004; 80: 257-63.

14. Stevens VJ, Obarzanak E, Cook NR, Lee IM, Appel LJ, Smith West D et al. Long term weight loss and changes in blood pressure: results of the trials in hypertention prevention. Phase II. Ann Intern Med 2001; 134: 1-11.

15. Goldstein DJ. Beneficial effects of modest weight loss. Int J Obes 1992; 16: 397-415.
16. Van GaAl LF, Wauters MA, De Leeuw IH. The beneficial effects of modest weight loss on cardiovascular risk factors. Int J Obes 1997; 21 (Suppl 1): S5-S9.

17. Tuomilehto J, Lindstrom J, Eriksson JG, Valle TT, Hamalainen H, Ilanne-ParikKa P et al. Prevention of type 2 diabetes mellitus by changes in lifestyle among subjects with impaired glucose tolerance. N Engl J Med 2001; 344: 1343-50.

18. Knowler WC, Barrett-Connor E, Fowler SE, Hamman RF, Lachin JM, Walker EA et al; Diabetes Prevention Program Research Group. Reduction in the incidence of type 2 diabetes with lifestyle intervention or metformin. N Engl J Med 2002; 346: 393-403.

19. The Diabetes Prevention Program Research Group. Impact of intensive lifestyle and metformin therapy on cardiovascular disease risk factors in the diabetes prevention program. Diabetes Care 2005; 28: 888-94.

20. Matthews DR, Hosker JP, Rudenski AS, Naylor BA, Treacher DF, Turner RC. Homeostasis Model Assessment: insulin resistance and B-cell function from fasting plasma glucose and insulin concentrations in man. Diabetología 1985; 28: 412-9.

21. Mcguire M, Wing R, Hill J. Prevalence of weight loss maintenance among American adults. Int J Obes 1999; 23: 1314-9.

22. WING RR, Phelan S. Long-term weight loss maintenance. Am J Clin Nutr 2005; 82 (1 Suppl): 222S-225S.

23. Moreno M, Manrique M, Guzmán S, Maiz A, Patino C, VAldés R ET AL. Cambios en los factores de riesgo metabólicos en pacientes obesos en tratamiento. Rev Méd Chile 2000; 128: 193-200.

24. Norris St, Zhang X, Avenell A, Gregg E, Schmid Ch, LAU J. Long-term non pharmacological weight loss interventions for adults with prediabetes. Cochrane Database Syst Rev 2005; Issue 2: CD005270.

25. Shaw K, O'rourke P, Del Mar C, Kenardy J. Psychological interventions for overweight or obesity. Cochrane Database Syst Rev 2005; Issue 2: CD003818.

26. Ministerio de Salud, Gobierno de Chile. Resumen Ejecutivo: Objetivos Sanitarios para la década 2000-2010. http://epi.minsal.cl/epi/html/elvigia/ vigia15.pdf (Consultado el 19 de febrero de 2007).

27. Teixeira PJ, Going SB, Sardinha LB, Lohman TG. A review of psychosocial pre-treatment predictors of weight control. Obes Rev 2005; 6: 43-65.

28. Elfhag K, Rössner S. Who succeeds in maintaining weight loss? A conceptual review of factors associated with weight loss maintenance and weight regain. Obes Rev 2005; 6: 67-85. 